\title{
APPLICATIONS OF THE ERLANG B AND C FORMULAS TO MODEL A NETWORK OF BANKING COMPUTER SYSTEMS - MOVING TOWARDS GREEN IT AND PERFORMANT BANKING
}

\author{
Florin-Catalin ENACHE and Adriana-Nicoleta TALPEANU \\ The Bucharest University of Economic Studies, Bucharest, Romania
}

\begin{abstract}
This paper surveys the contributions and applications of queueing theory in the field of banking data networks. We start by highlighting the history of IT and banks and we continue by providing information regarding the main prudential regulations on the banking area as Basel Accords and green IT regulations, that on one side generate more computing needs and on the other side promote conscientious use of the existing IT systems.

Continuing with a background of the network technologies used in Economics, the focus will be on the queueing theory, describing and giving an overview of the most important queueing models used in economical informatics. While the queueing theory is characterized by its practical, intuitive and subtle attributes, the queueing models are described by a set of 3 factors: an input process, a service process and a physical configuration of the queue or the queueing discipline.

The Erlang $B$ and $C$ mathematical definitions of formulas for a specific number of servers, at the $\lambda$ arrival rate, and the average service time will be described, used and confirmed by computer simulations of real queues usually found in the banking computing systems.

The goal is to provide sufficient information to computer performance analysts who are interested in using the queueing theory to model a network of banking computer systems using the right simulation model applied in real-life scenarios, e.g. overcoming the negative impacts of the European banking regulations while moving towards green computing.
\end{abstract}

\section{KEYWORDS}

queueing theory, banking system, Erlang B, Erlang $C$, computer network, economical informatics, banking regulation, computer simulation, Basel.

\section{INTRODUCTION}

From the first recorded bank in the world, Taula de la Ciudad, which opened in Barcelona in 1401, to the current known banks, the services provided by banks developed considerably. The Bank Taula de la Ciudad was founded as a treasury resource for the Catalonian government. Even if the bank is on record as the first official bank in the world, the practice of banking has been traced back for several centuries. [12]

Jan Zizka et al. (Eds) : CCSEIT, AIAP, DMDB, MoWiN, CoSIT, CRIS, SIGL, ICBB, CNSA-2016

pp. 23-39, 2016. () CS \& IT-CSCP 2016

DOI : $10.5121 /$ csit.2016.60603 
Many histories position the crucial historical development of a banking system to medieval and Renaissance Italy and particularly the affluent cities of Florence, Venice and Genoa. The Bardi and Peruzzi families dominated banking in 14th century Florence, establishing branches in many other parts of Europe. [7]

Perhaps the most famous Italian bank was the Medici bank, established by Giovanni Medici in 1397. [6] The oldest bank still in existence is Monte dei Paschi di Siena, headquartered in Siena, Italy, which has been operating continuously since 1472. (Boland, 2009).

The banking development spreads from northern Italy throughout the Holy Roman Empire, and to northern Europe in the 15th and 16th century. Another important point in time is the development of the important innovations that took place in the 17th century, in Amsterdam, during the Dutch Republic, and in the 18th century, in London. Of course, the development heavily continues with the innovations in telecommunications and computing, in the 20th century, when the size of the banks and the geographical coverage increase, due to the development of the operations' side of the banks. During the well-known financial crisis from 2007-2008, all the banks were affected, some of them more than others, causing a specific attention to the banking regulations in the upcoming years.

On the IT side, even if usually most observers prefer and are expected to discuss about what is coming and not about what happened, we would like to highlight at least the three most important events in the IT history. The first event may be considered the document "First Draft of a Report on the EDVAC" published by John Von Neumann end of June 1945, consisting of the first documented discussion of the stored program concept and the blueprint for computer architecture to this day [13]. It is also called "the technological basis for the worldwide computer industry" [1]. The second important event may be considered the giving birth of the Ethernet, by Bob Metcalfe, in 1973, at the Xerox Palo Alto Research Center (PARC). The third event is in 1989, when Tim Berners-Lee circulated "Information management: A proposal" at CERN in which he outlined a global hypertext system.

\subsection{Banking and IT regulations}

All types of banks, being part of the banking system, have to comply with the banking regulations. It can be distinguished three classes of banking regulations: economic regulation, prudential regulation and monetary regulation. What we will deepen further is the prudential or prevented regulation, which is designed "to ensure efficient allocation of resources, to minimize the risks assumed by banks and to ensure stability and financial soundness of individual banks and of the banking system as a whole". [3]

On the IT side, the regulation topic become more and more discussed during the recent years due to the huge development of the IT world.

\subsection{Basel Banking Accords and other banking regulation}

Nowadays, the most important international banking regulations are the Basel Accords. These accords are published in Basel, Switzerland, by the Bank for International Settlements BIS in Basel, which is the central body in charge to develop and standardize banking regulations. 
Basel I was the first accord adopted in 1988 and had the objective to improve the equity of internationally active banks by establishing a relation between equity and risk-weighted assets. Thus, Basel I proposed a standard methodology for calculating the equity and two solvability indicators to ensure compliance with the minimum coverage of risky assets (net exposure) through the bank capital. AT the end, it was concluded that the first indicator is enough for satisfying the minimum level of the solvability ratio 1 and the net exposure was calculated based on the credit risk, considering four risk categories $(0 \%, 20 \%, 50 \%$ and $100 \%)$, applied according to the category of considered assets [11].

Basel II was adopted in 2004 and had the objective to cover various complains followed by Basel I. This accord contains changes to supervisory, regulatory and international cooperation between various authorities, his objectives being organised in three pillars: Pilar 1, Pilar 2 and Pilar 3. [3] Pillar 1, also referred as "Minimum Capital Requirements", contains minimum capital requirements for credit risk, market risk and operational risk.

Pillar 2, also referred as "Supervisory Review Process", covers a qualitative approach about prudential requirements through the supervisory process. In addition to the risk defined in the Basel I, in Basel II the following risks are covered: the liquidity risk, residual risk, strategic risk, reputational risk, concentration risk and interest rate risk for exposures which are not in the trading book.

Pillar 3, also referred as "Market discipline", offers to the shareholders and the investors the possibility to monitor more effectively the bank management, because it requires to the banks to develop a set of detailed reporting requirements for the supervisory authority and for the public.

Basel III was issued as a result of the well-known global financial crisis, from 2007 and is improving several aspects of Basel II, a visual comparison being visible in the Figure 1. This accord requires from banks to have more equity of a superior quality, in order to be prepared to the future crisis, using Capital "Requirements Directive CRD IV" and "Capital Requirements Regulation CRR". In addition, this accord defines a minimum leverage of $3 \%$ and two mandatory liquidity ratios: the rate of immediate liquidity and the long-term liquidity ratio. It is also enhancing the supervisory review process for firm-wide risk management and capital planning and the risk disclosure and the market discipline.

Worldwide, are currently existing also some other financial development institutions that took the role of supporting financial environment to adapt to a changing word. One of the largest ones is the International Finance Corporation (IFC), which hosts an informal group of banking regulators and banking associations, called Sustainable Banking Network. The group is currently designed to help regulatory authorities of emerging markets to develop green credit policies and environment and social risk management guidelines by sharing knowledge and technical resources. At the moment, the network has members from Bangladesh, Brazil, China, Indonesia, Lao PDR, Mongolia, Nigeria, Peru, Thailand and Vietnam. 


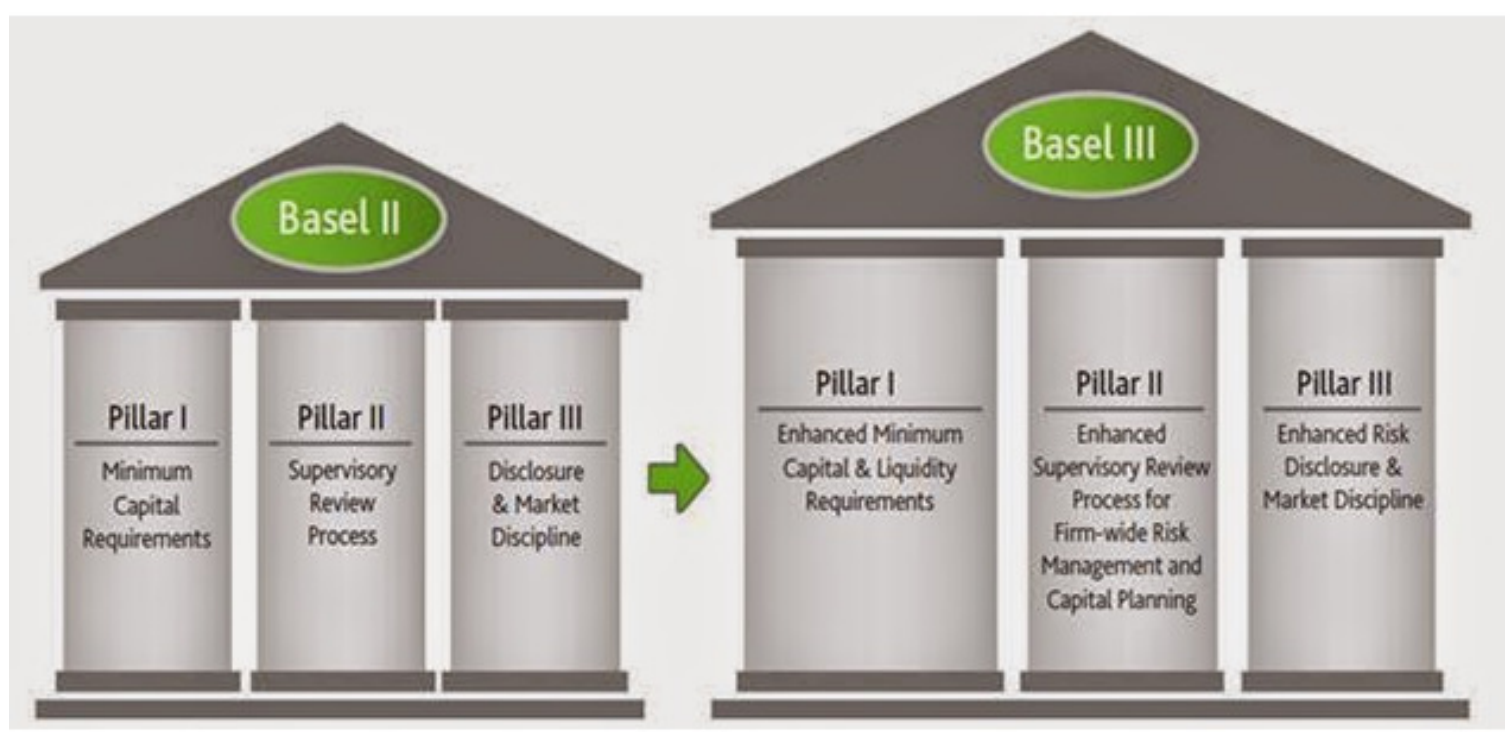

(Source: Srivastava, A., 2016)

Fig. 1: Comparison between Basel II and Basel III

\subsection{Green computing legislation}

One of the first initiatives was taken place in USA, in 1992, and it was named Energy Star. This was a voluntary labelling program, created by the Environmental Protection Agency, having the purpose to promote energy efficiency in hardware components. In the recent years, the awareness about the necessity of a Green computing was increasing and therefore more directives appeared.

For example, the first WEEE Directive (Directive 2002/96/EC) entered into force in early 2003. WEEE stands for waste of electrical and electronical equipment such computers, TV-sets, fridges and cell phones. The directive has to goal to increase the recycling of WEEE and/or its reutilisation, by creating different collection schemes of WEEE, free of charge for population. [17]

The EU legislation (RoHS Directive 2002/95/EC) has to scope to restrict the use of hazardous substances in electrical and electronical equipment, referring specially to the heavy metals as mercury, lead, flame-retardants, cadmium, and why not, to find a cheaper and not that noxious substitutes. A newly revised directive by the European Commission became effective beginning of 2012. [17]

\section{NETWORK TECHNOLOGIES USED IN ECONOMICS}

With the global advancement seen in the last 20 years, especially with the increased volume, complexity, spread of exchanges in the economic and financial relations, all the computing systems, but especially the banking systems, had to adapt fast not only their banking regulations [16] [18] to the continuous changing world [12] but also their networking field, which had changed drastically over the time. Perhaps, the most fundamental change has been the rapid development of optical fiber technology. This has created limitless opportunities for new digital networks with greatly improved capabilities. The current broadband integrated service networks that provide integrated data, voice and video seem to have almost nothing in common with the 
data networks of the last 20 years, but in fact, many of the underlying principles, mathematical and statistical laws are the same.

\section{QUeueing Theory BACKgrounds - Probability, Stochastic PROCESSES AND MATHEMATICS}

Probability is a beautiful field of mathematics that is rich in its depth of deductive reasoning and in its diversity of applications. With its roots in the 17th century, probability started with simple counting arguments that were used to answer questions concerning possible outcomes of games of chance. Over the centuries, probability has been established as a key tool in a wide range of diverse fields like biology, chemistry, computer science, finance, medicine, physics, etc.

Probability served as the basis for deriving results to study stochastic processes. A stochastic process can be thought of as being a set of outcomes of a random experiment indexed by time. As an example, $X_{n}, n=1,2,3, \ldots$, could be the total number of tails obtained from the first $\mathrm{n}$ tosses of a fair coin in an experiment that continues for an indefinite period of time. The following set, $\left\{X_{1}, X_{2}, X_{3}, \ldots,\right\}$ represents a process to indicate that there is a relationship or dependency between the random variables $X_{n}$ [5]. For continuous time processes, $X(t)$ is a stochastic process and the values of $X(t)$ and $X\left(t^{\prime}\right)$, for $t<t^{\prime}$ have some kind of relationship or dependency.

A frequent application area for probability and stochastic processes is the queueing theory. The nomenclature used in queuing applications can be easily explained by the terms in Fig.2:

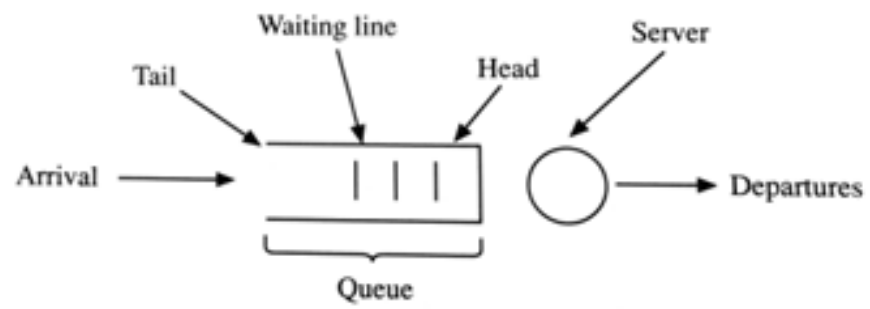

Fig.2. A single server queue

A single server queue consists of a server that processes customer requests, a waiting line or queue where customers wait before receiving service and an arrival stream of customers. For example, customers can arrive at the tail of the queue and are served on a first-come-first-served (FCFS or FIFO) basis [2]. In a computer model, the server could correspond to a hard drive that processes read or write requests, a CPU that processes customer requests or to a router serving a network of computers sending network requests. Typically, if the waiting room is finite, then any customer coming during the time when the waiting queue (the so called "buffer" in computer science terminology) is full is assumed to be lost to the system, just like if the customer never arrived.

The queuing theory is described by 3 fundamental characteristics:

- practical, as it has direct practical applications, for example, in modeling a network of computers in a banking or cloud environment 
- intuitive, making it simpler to generate real-life models.

- subtle, making it intellectually interesting, because it uses probability to describe reality.

When speaking about a real-life model, it is common to have a multiple server queue, especially in modern CPU systems. Customers arrive at random, and try to find a server. If a server is available, they take it and hold it for a random amount of time, named onwards service time. If not, one possibility is that the customers that arrive when all servers are busy overflow and are lost (Erlang B formula). It is also possible that these customers wait in a queue (Erlang C formula).

A queueing model is defined by its:

- input process - a random process that defines the way in which the customers arrive, which are represented by the arrows in Fig.3.

- $\quad$ service process - a random process that defines the length of the service times needed by the arriving customers, which may be seen graphically by the height of the black bars above the arrows in Fig.3.

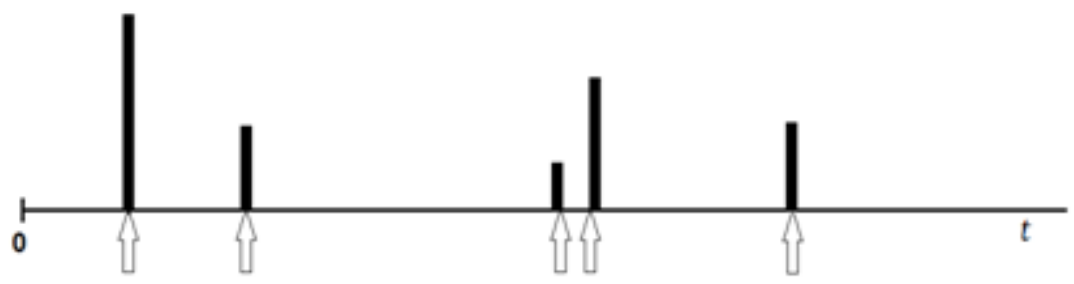

Fig.3. Random arrival with Random service times

- physical configuration or the queue discipline which defines what happens if a customer is blocked or waits in a queue. When the customers wait in a queue, more information is needed to describe the queue discipline, for example, the size of the queue, if a customer waits indefinitely or drops after a certain amount of time, the order in which a customer is served, e.g. last-come-first-served (LCFS) or in a random order etc.

\section{The ERlang B Formula - Probability OF BLOCKING}

For analysing this model, we are going to assume:

- $s$ servers

- requests that arrive at a certain arrival rate $\lambda$

- $\quad$ an average service time $\tau$

This queue will be intuitively analysed by using the classical rate-up=rate-down argument used in engineering, then the model will be limited and confirmed by using computer simulations.

The Fig. 3 below describes the system states for a queue with 3 services. Understanding the mechanism from Fig. 3 will help define a mathematical model and later develop the computer model simulation used to confirm the specific cases where this is available. 
For this specific system we could be in state $0,1,2$ or 3 , which represents the number of customers present in the system - $N(t)$. We start at state 0 , where no request is present in the system. Once the first customer arrives, the system jumps to state 1, it will stay there a while, until either that request completes or another request arrives. If another call arrives before the first one completes, then the system jumps to state 2 , and stays there a while. Consequently, jumps down to 1 , then it jumps up to state 2 , and then maybe to state 3 , and so one. If a customer arrives in state 3 , then because all servers are busy, that would be a lost request. Practically every jump up corresponds to an arrival and all the jumps down correspond to a departure. Rate up=rate down means in this case that on the long term the number of the customers that arrive will equal to the number of customers that leave the system.

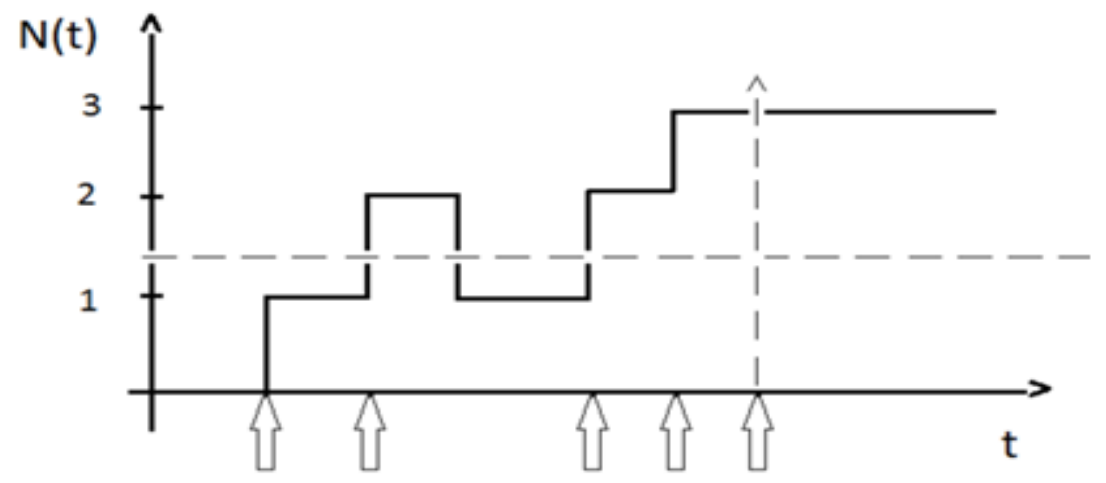

Fig.4. Random arrivals and random service times in a multi-server queue

The model will be analyzed by equating for each state the rate at which the system jumps up from that state to the rate the system jumps down from the state above it. If we look at the Fig.4., the dotted line shows that the number of jumps up and the number of jumps down will differ by at most 1 , which in limit does not matter. That means that by dividing the number of jumps up by the total amount of time we get the rate up, and by dividing the number of jumps down by the total amount of time results the rate down. The rate up from $P_{0}=\lambda P_{0}=\frac{1}{\tau} P_{1}=$ rate down from $P_{1}$. By the same argument, $\lambda P_{1}$ would be the rate up from state 1 , and $\frac{2}{\tau} P_{2}$ is the rate down from state 2 , since from state 2 there are 2 chances for a completed request. Going further with this argument, the following set of equations is developed:

$$
\begin{aligned}
& \lambda P_{0}=\frac{1}{\tau} P_{1} \\
& \lambda P_{1}=\frac{2}{\tau} P_{2} \\
& \lambda P_{2}=\frac{3}{\tau} P_{3} \\
& \mathrm{M} \\
& \lambda P_{s-1}=\frac{s}{\tau} P_{s}
\end{aligned}
$$


The problem is reduced to solving this set of equations and finding $P_{s}$ - the probability that all servers are busy. By normalizing and using the notation $\lambda \tau=a$, it is hints that the solution would depend on the number of servers and on the product $\lambda \tau=a$, called offered load, and not by the individual values of either $\lambda$ or $\tau$.

$$
\begin{aligned}
& P_{1}=\lambda \tau P_{0}=a P_{0} \\
& P_{2}=\lambda \tau P_{1}=\frac{a^{2}}{2} P_{0} \\
& P_{3}=\lambda \tau P_{2}=\frac{a^{3}}{3 !} P_{0} \quad \Rightarrow P_{j}=\frac{a^{j}}{j !} P_{0}, j=1,2, \ldots, s
\end{aligned}
$$

M

$$
P_{s}=\lambda \tau P_{s-1}=\frac{a^{s}}{s !} P_{0}
$$

The set of equations is completed by the fact that:

$$
\begin{aligned}
& P_{0}+P_{1}+\ldots+P_{s}=1 \Rightarrow P_{0}+\frac{a^{1}}{1 !} P_{0}+\frac{a^{2}}{2 !} P_{0}+\ldots+\frac{a^{s}}{s !} P_{0}=1, \text { therefore, } \\
& P_{0}=\frac{\frac{a^{s}}{s !}}{1+\frac{a^{1}}{1 !}+\frac{a^{2}}{2 !}+\ldots+\frac{a^{s}}{s !}} \text {, resulting that } P_{s}=\frac{\frac{a^{1}}{1+\frac{a^{2}}{1 !}+\ldots+\frac{a^{s}}{s !}}}{2 !} E_{1, s}(a)=B(s, a)
\end{aligned}
$$

also named the Erlang B formula, giving the probability of blocking, which is the probability that all servers are busy.

At this moment, it is quite easy to calculate how many servers would be needed in order to have, let's say less than $1 \%$ dropped requests. This is important on both financial and quality aspects. If too many servers are provided, then the service will be good, but the entire system will be more expensive than necessary. Looking at Fig. 5, we would draw the line corresponding to 0.01, estimate the offered load, and then search for the first curve that would lie below point of intersection. This points out the practical application of the Erlang B formula that helps engineers to calculate the number of servers needed for given values of the offered load and percent of lost requests.

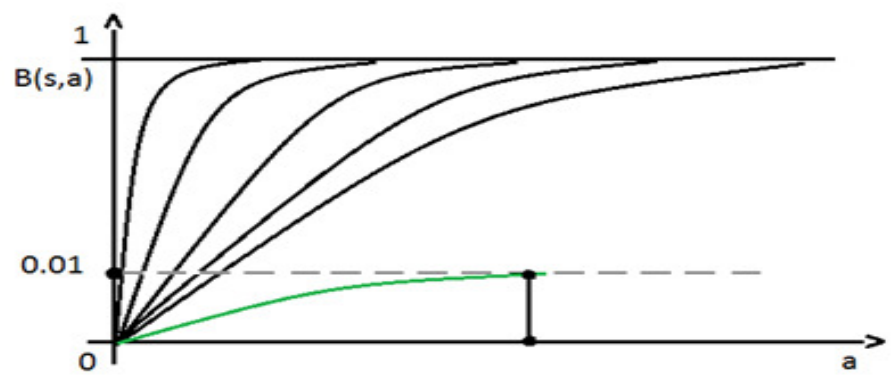

Fig.5. Erlang B graph 


\section{SUBTLETIES OF THE ERLANG B FORMULA - COMPUTER SIMULATIONS}

Looking at Fig.6 we can observe the following arrival processes that have the same rate of arrival $\lambda$ :

- constant arrival process - green

- random arrival process - black

- in between arrival process - red

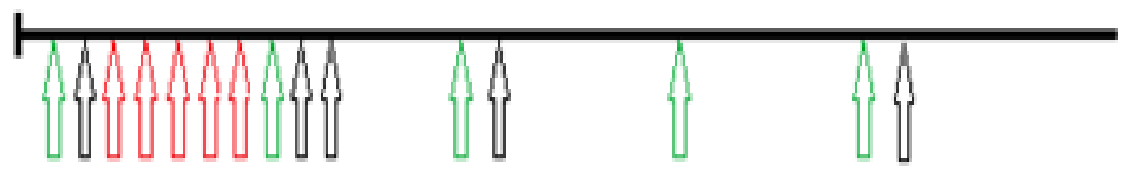

Fig.6. Different types of arrival processes

The question is which type of arrival process is described by the previous rationale. The rationale could be always right, never or just sometimes (Poisson exponential arrivals). Also this could be extended to the service process. To easily demonstrate that, a computer simulation will be developed.

The physical interpretation of $\mathrm{B}(s, a)=20 \%$ is one of the subtleties of the Erlang B formula. Is it that $20 \%$ of all requests are going to be lost? Another possibility is that $20 \%$ of the time all servers are busy. Further, is another question arises: what exactly is $P_{2}$ - the probability of being in state 2? Is that the fraction of arrivals who finds the system in state 2 , or the fraction of time the system is in state 2 ?

The following computer code written in BASIC will calculate the fraction of time the system is in the blocking state (a ratio of times) and the fraction of customers who arrive during the blocking state (ration of integers - number of customers that overflow divided by the total number of customers who arrive). So the left and the right sides of the (1) equations are measured in complete different ways. The computer code assumes the arrival rate to be 4 , the average service times to be 2.4 resulting an offered load of $4 \times 2.4=9.6$ erlangs, and the number of servers to be 10 . The simulation will run for 100000 arriving requests, and the types of arrival and service time processes will be considered as follows:

- Poisson arrivals, exponential service times

- Poisson arrivals, constant service times

- Constant inter-arrival times, exponential service times

- Constant inter-arrival times, constant service times 


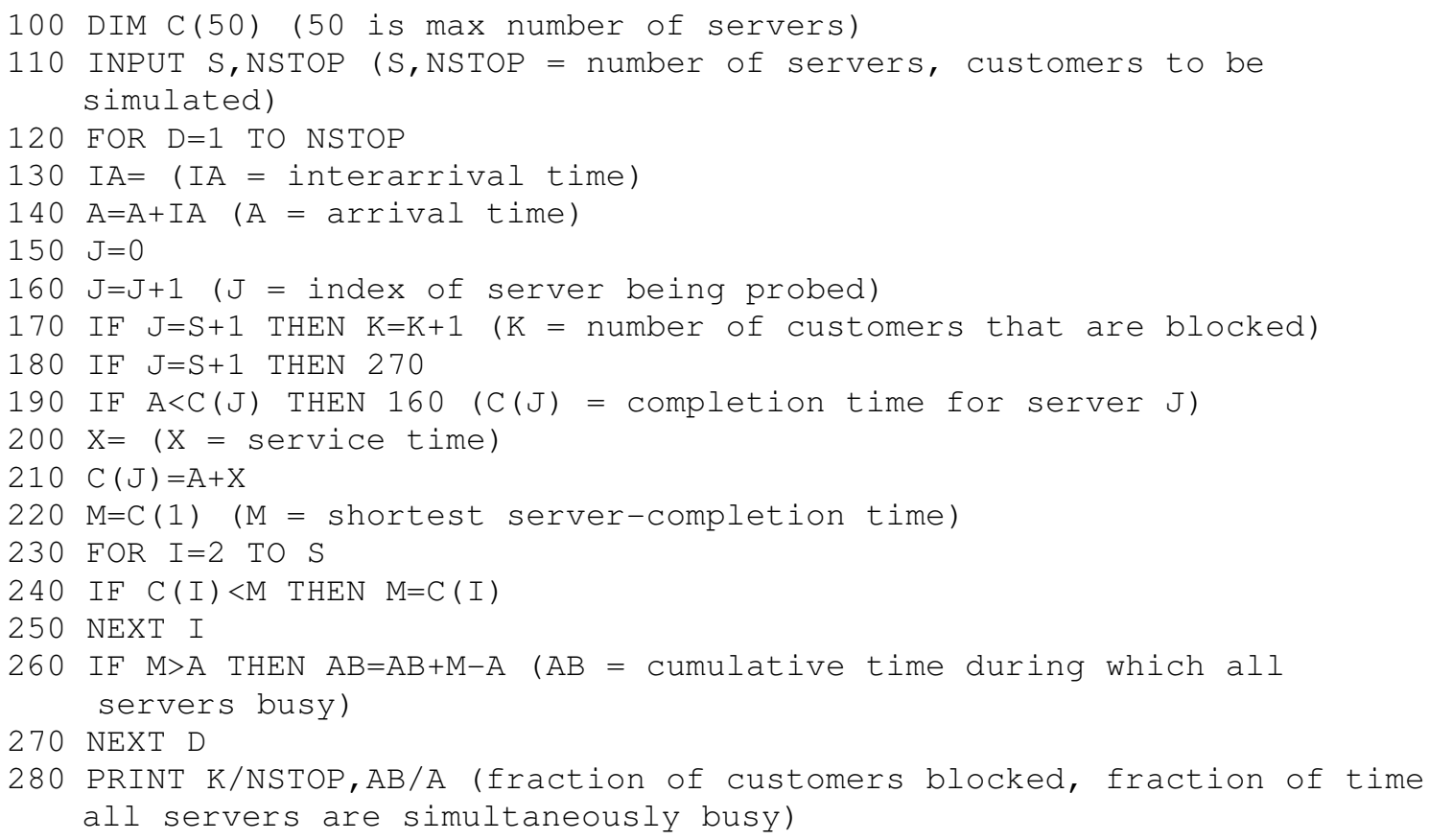

\section{SIMULATION INPUTS AND RESULTS FOR ERLANG B}

Table 1. Erlang B - Simulation inputs and outputs

\begin{tabular}{|c|c|c|c|c|c|c|}
\hline & IA [10] & $\mathrm{X}$ & K/NSTOP & $\mathrm{AB} / \mathrm{A}$ & \multicolumn{2}{|c|}{$\mathrm{B}(s, a)$} \\
\hline 1 & $-(1 / 4)^{*} \mathrm{LOG}(1-\mathrm{RND})$ & $-2.4^{*} \mathrm{LOG}(1-\mathrm{RND})$ & 0.19652 & 0.1958451 & \multirow{2}{*}{\multicolumn{2}{|c|}{$19.6 \%[16]$}} \\
\hline 2 & $-(1 / 4)^{*}$ LOG $(1-R N D)$ & 2.4 & 0.19652 & 0.1958451 & & \\
\hline 3 & $1 / 4$ & $-2.4^{*} \mathrm{LOG}(1-\mathrm{RND})$ & 0.13366 & .2360997 & N/A & $\mathrm{N} / \mathrm{A}$ \\
\hline 4 & $1 / 4$ & 2.4 & 0 & 0.6009062 & $\mathrm{~N} / \mathrm{A}$ & $\mathrm{N} / \mathrm{A}$ \\
\hline
\end{tabular}

As seen in Table 1, the variability in the service times does not affect the answer, but the variability in the arrival process does.

No matter what distribution function we use to describe service times, the answer is unaffected. From a practical point of view, something that is difficult to measure, does not have to be measured because the final answer does not depend on it. We can completely disregard the statistical characteristics of the service times, once this model is understood. That is why these formulas are so robust and safe to use.

\section{THE ERLANG C FORMULA - BLOCKED CUSTOMERS DELAYED}

By extending the heuristic conservation-of-flow to include the case in which all customers who find all servers busy wait until they are served, and by following the same intuitive approach, rate up=rate down, we get the following set of formulas: 


$$
\begin{aligned}
& \lambda P_{0}=\frac{1}{\tau} P_{1} \\
& \lambda P_{1}=\frac{2}{\tau} P_{2} \\
& \lambda P_{2}=\frac{3}{\tau} P_{3} \quad \Rightarrow P_{j}=\frac{a^{j}}{j !} P_{0}, j=0,1, \ldots, s-1
\end{aligned}
$$

M

$$
\lambda P_{s-1}=\frac{s}{\tau} P_{s}
$$

Further, when looking at the first set of formulas it was concluded that the rate down from state 2 is $\frac{2}{\tau}$, but at the point where all servers are busy, the aggregate service completion rate would be constant and equal to $\frac{s}{\tau}$ because all servers are busy, and only $s$ customers are served. This leads to the following set of equations:

$$
\begin{aligned}
& \lambda P_{s}=\frac{s}{\tau} P_{s+1} \\
& \lambda P_{s+1}=\frac{s}{\tau} P_{s+2} \\
& \lambda P_{s+2}=\frac{s}{\tau} P_{s+3} \quad(5) \Rightarrow \begin{array}{l}
P_{s+1}=\frac{a}{s} P_{s}=\frac{a^{s+1}}{s ! s^{1}} \\
\mathrm{M}
\end{array} P_{s+2}=\frac{a^{s+2}}{s ! s^{2}}
\end{aligned}
$$

By combining equations (4) and (5), the following formula is deducted for this model:

$$
P_{j}=\left\{\begin{array}{l}
\frac{a^{j}}{j !} P_{0},(j=1,2, \ldots, s-1) \\
\frac{a^{j}}{s ! s^{j-s}},(j=s, s+1, \ldots)
\end{array}\right.
$$

By normalization (requirement that all probabilities add up to 1) we get:

$$
\begin{aligned}
& P_{0}+P_{1}+\ldots+P_{s}+P_{s+1}+\ldots=1=>P_{0}\left(1+\frac{a^{1}}{1 !}+\frac{a^{2}}{2 !}+\ldots+\frac{a^{s-1}}{\left(s \overline{4}_{4} 1\right) !}+\frac{a^{s}}{s_{4} !}+\frac{a^{s+1}}{2^{s ! s^{1}}{ }_{4}}+\frac{a^{s+2}}{s^{!} s_{4}^{2}}+\ldots 3 \text { 43 }=1\right. \\
& \frac{a^{s}}{s !}\left(1+\left(\frac{a}{s}\right)^{1}+\left(\frac{a}{s}\right)^{2}+\ldots\right)
\end{aligned}
$$

It is obvious that the right part of the above equation is an infinite geometric series and the formula only makes sense if the series converges. By mathematical reasoning, the series 


$$
\begin{aligned}
& \text { converges to } \frac{1}{1-\left(\frac{a}{s}\right)} ; \text { therefore, the formula makes sense if and only if } a<s \text {. We conclude that } \\
& P_{0}=\frac{1}{\sum_{k=0}^{s-1} \frac{a^{k}}{k !}+\frac{a^{s}}{s !\left(1-\frac{a}{s}\right)}} \quad(8), \quad \text { if } \frac{a}{s}<1
\end{aligned}
$$

\section{SubTLETIES OF THE ERLANG C FORMUla}

Since $\frac{a}{s}$ must be less than 1 , then $a$ has to be less than $s$, it results that $\lambda \tau<s$, therefore $\lambda<\frac{s}{\tau}$. This means that the arrival rate must be less than the maximum average completion rate, otherwise the queue is going to grow to infinity, being impossible to find an equilibrium state. The Erlang B formula calculates the probability of the system to be in the blocking state, which is equal to the fraction of customers who find the blocking state and therefore are lost. In the case of the Erlang $\mathrm{C}$ formula, the fraction of time when the system finds itself in the blocking state is calculated, but in this case, the blocking state (probability of queueing) means not only state $s$, but also state $s+1, s+2$, etc., while limiting this rationale to Poisson input.

$$
\begin{aligned}
& C(s, a)=P_{s}+P_{s+1}+P_{s+2}+\ldots=E(2, a) \text { (by definition) } \\
& C(s, a)=P_{s}+P_{s+1}+P_{s+2}+\ldots=P_{0} \frac{\frac{a^{s}}{s !}}{a-\left(\frac{a}{s}\right)}=\frac{\frac{a^{s}}{s !\left(1-\frac{a}{s}\right)}}{\sum_{k=0}^{s-1} \frac{a^{k}}{k !}+\frac{a^{s}}{s !\left(1-\frac{a}{s}\right)}}=E_{2, s}(a)=\frac{s B(s, a)}{s-a(1-B(s, a)}
\end{aligned}
$$

(9), giving the fraction of customers who have to wait in the queue in a model in which all blocked customers wait as long as necessary to be served.

Getting back to the rate-up=rate-down assumption, the following is true:

$$
\lambda P_{j}=\mu_{j+1} P_{j+1}, \text { where } \mu_{k}=\left\{\begin{array}{l}
\frac{k}{\tau},(k<s) \\
\frac{s}{\tau},(k \geq s)
\end{array}\right.
$$

When considering the rate-up=rate-down argument, how long the system is in any particular state was not taken into consideration, but only that the system is in that state. The rate at which the system goes up from state $j$ depends only on $j$ - the number of customers present - , but it does not depend on the past history of the system, except that that the past history produces this current state. Likewise, if the system is in state $j+1$, the rate at which the system goes down (rate at which 
customers leave the system) depends only on how many customers are currently present and not on the past history of the system. In other words, it does not depend on when the last arrival occurred, it depends on how much service remains for each of the customers who are being served. By using the rate-up=rate-down argument, the past history is neglected, and this implies that the underlying variables are exponential, because of the Markov property [9], which states that the only thing that affects the future evolution of a random variable that is exponential is its basic parameters but not how long it has been in progress. This is also an intuitive interpretation as to why a sufficient condition to this rationale is that all the underlying variables are exponential. One exception to this rule was analyzed earlier under the Erlang B formula when the blocked customers are cleared. The requirement for this is that we have Poisson input and exponential service times, where for Erlang B having Poisson arrivals but not necessarily exponential service times was enough.

In Fig.4, for the Erlang B as the offered load increases, the probability of blocking increases and is asymptotic to 1 , getting a sequence of curves. For an increasing number of servers, the curve for a larger number of servers $s$ lies below the curve for a smaller number of servers, because the larger number of servers will reduce the probability of blocking for the same value of $a$ - offered load.

By plotting the Erlang $\mathrm{C}$ values, the result is similar, but not exactly the same, because now we have the condition that $a$ must be less than $s(a<s)$. For 1 server, when $a$ is equal to 1 erlang, the curve would not be asymptotic to 1 , but it will reach 1 , because when the offered load is 1 or more, it means that the infinite series does not converge and the probability of waiting in a queue is $100 \%$. Redoing the before mentioned thinking, we get the following graph:

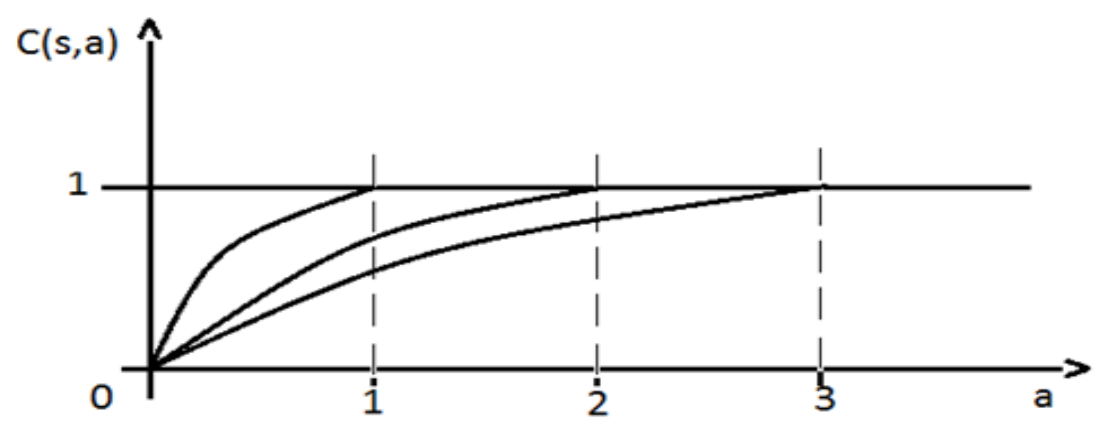

Fig.7. Erlang C graph

The model used in the Erlang $\mathrm{C}$ simulation is called M/M/s by the conventional queueing theory notation. The general model introduced by Kendall [19] in 1953 can be summarized as follows: $a / b / c$, where:

- $a$ indicates the arrival process, where M represents the memoryless Markov property Poisson input

- $b$ indicates the service process, where M represents Markov memoryless property exponential service times

- $\quad c$ is the number of servers, and it is implicitly that there is an infinite queueing capacity 
The Erlang B model is by the same notation M/G/s/s, having Poisson input, general service times, $s$ servers and the $s$ capacity of the system (no waiting positions). Further details on the M/G/1 [9] model, including a simulation can be found in the reference [4].

The following computer code written in BASIC will simulate the same problem as with the Erlang B case, with the only difference that the blocked customers are now allowed to wait in a queue. The simulation will calculate what the customers see, more exactly the fraction of customers who have to wait in the queue, which is equal to the fraction of time the system is blocked, according to the PASTA Theorem [14]. The computer code assumes the arrival rate to be 4 , the average service times to be 2.4 resulting an offered load of $4 \times 2.4=9.6$ erlangs, and the number of servers to be 10 . The simulation will be run for 100000 arriving requests, and the types of arrival and service time processes will be considered as follows:

- Poisson arrivals, exponential service times. (M/M/s)

- Poisson arrivals, constant service times. (M/D/s)

- Constant inter-arrival times, exponential service times. (D/M/s)

- Constant inter-arrival times, constant service times. (D/D/s)

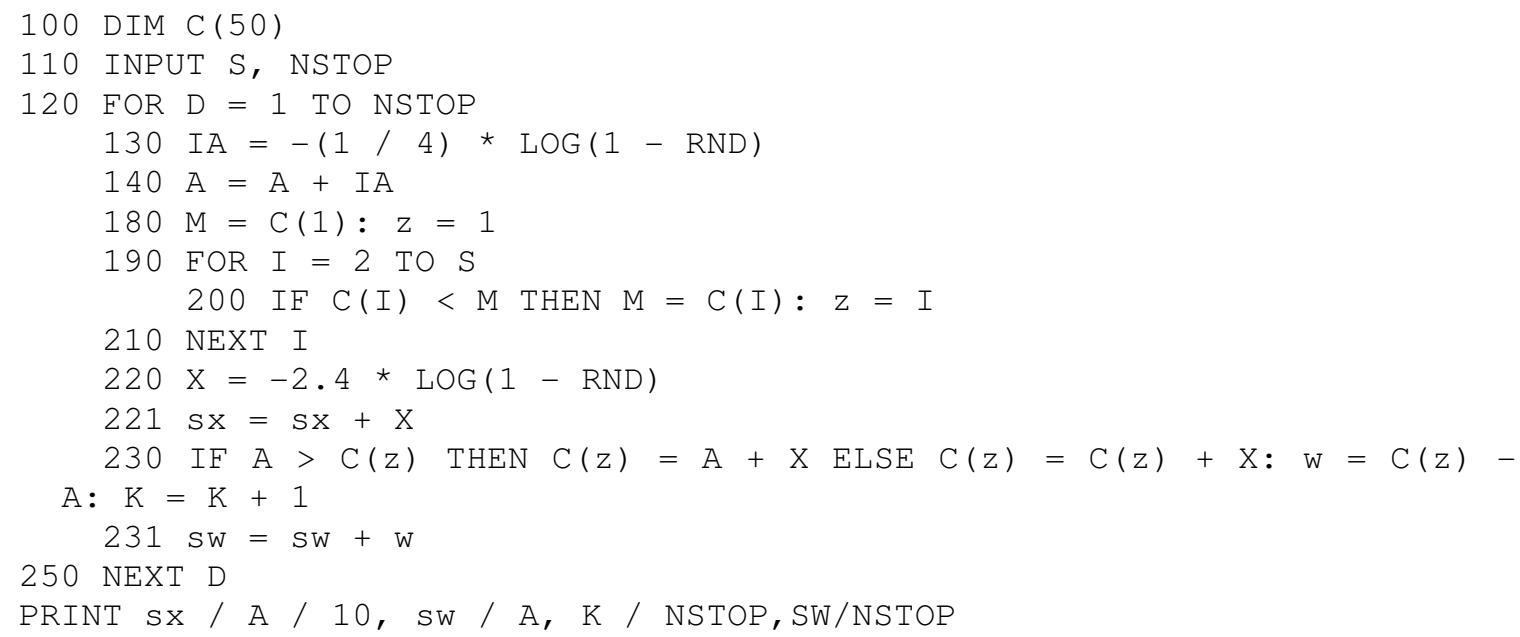

\section{SimUlATION INPUTS AND RESULTS FOR ERLANG C}

Table 2. Erlang $\mathrm{C}-$ Simulation inputs and outputs

\begin{tabular}{|l|l|l|l|l|l|l|l|l|}
\hline $\mathrm{s}=10$ & \multicolumn{2}{|c|}{ l } & \multicolumn{2}{c|}{$\mathbf{2}$} & \multicolumn{2}{c|}{$\mathbf{3}$} & \multicolumn{2}{c|}{4} \\
\hline$\lambda=4$ & Theory & simulation & Theory & simulation & Theory & simulation & Theory & simulation \\
\hline$\rho=\frac{a}{s}$ & $96 \frac{8}{8}$ & 0.9650543 & $96 \frac{8}{8}$ & 0.9704734 & 968 & 0.9497337 & $96 \frac{8}{8}$ & 0.9600935 \\
\hline $\begin{array}{l}E(W)=\frac{C(s, a)}{(1-\rho) s} \tau \\
{[8]}\end{array}$ & 6.45762 & 6.469176 & N/A & 5.999352 & N/A & 4.222098 & 0 & 0 \\
\hline$P(W>0)=C(s, a)$ & 0.859046 & 0.85792 & N/A & 0.8586 & N/A & 0.7386 & 0 & 0 \\
\hline
\end{tabular}




\section{CONCLUSiOnS}

Considering the facts presented in the paper, the environment, the IT and the banking systems should not be seen as single independent entities and the strong existing interlinkage between all of them should be considered. Currently, are already existing few regulations that are connecting the environment with the IT and the banking systems and some initiatives are planned for the upcoming years, but the maturity is not that high.

By using the Erlang formulas, we are able to use the above stated probabilities to represent both the point of view of an outside observer simply by looking at a system over the total time and calculating these probabilities as a fraction of time, and the point of view of the arriving customers who see the system only at the instance at which they arrive, meaning finding the system in blocking state and therefore waiting in a queue.

For the Erlang B formula, the variability in the service times does not affect the answer, but the variability in the arrival process does. No matter what distribution function we use to describe service times, the answer is unaffected. From a practical point of view, something that is difficult to measure, does not have to be measured because the final answer does not depend on it. We can completely disregard the statistical characteristics of the service times, once this model is understood. This is the reason why these formulas are so robust and safe to use.

Based on all information presented in this paper, we can conclude that computer simulation is an important tool for the analysis of queues whose service times have any arbitrary specified distribution. In addition, the theoretical results for the special case of Poisson arrivals and exponential service times are extremely important because they can be used to check the logic and accuracy of the simulation, before extending it to more complex situations.

Moreover, such a simulation gives insight on how such a queue would behave as a result of different arrival processes and service times. Further, we consider that it offers a methodology for looking into more complicated cases not only like getting input times from a network of banking systems trying to implement a new set of banking regulations where a mathematical approach cannot help, but also in other complex areas.

Another important point of the paper is to have mandatory environmental enriched IT and banking regulations applicable worldwide, not only to some regions or countries as it is happening today. In this way, the regulators will be challenged to not think in silos anymore, on a short term, and to adopt a broader view applicable for a long term period, because everything is connected and the environmental impacts are affecting the entire world.

\section{REFERENCES}

[1] Campbell-Kelly, M., \& Aspray, I. (2004). Computer: A History Of The Information Machine (Sloan Technology). Westview Press

[2] Cooper, R. B. (1981). Introduction to Queueing Theory, Second Edition. New York: North Holland, New York.

[3] Dardac, N., Moinescu, B. (2007). Politici monetare și tehnici bancare. Course notes. 
[4] Enache, F.C. (2014). Stochastic Processes and Queueing Theory for Cloud Computer Performance Analysis, In: Conference Proceedings of the 14th International Conference on Informatics in Economy, pp13-19.

[5] Ghahramani, S. (2005). Fundamentals of Probability with Stochastic Processes, Third Edition. Upper Saddle River, Pearson Prentice Hall, New Jersey.

[6] Goldthwaite, R. A. (1995). Banks, Places and Entrepreneurs in Renaissance Florence: 492 (Variorum Collected Studies), Variorum.

[7] Hoggson, N. F. (1926). Banking Through the Ages. Dodd, Mead \& Company.

[8] Lakatos, L. (2008). A note on the Pollaczek-Khinchin Formula. In: Annales Univ. Sci. Budapest, Sect. Comp. 29, pp. 83-91.

[9] Sigman, K.. (2011). Exact Simulation of the stationary distribution of the FIFO M/G/c Queue. J. Appl. Spec. Vol. 48A, pp. 209-213, <http://www.columbia.edu/ ks20/papers/QUESTA-KSExact.pdf>. [January 20, 2015].

[10] Sigman ,K.. (2010). Inverse Transform Method, <http://www.columbia.edu/ ks20/4404Sigman/4404-Notes-ITM.pdf>. [January 15, 2015].

[11] Tarullo, D. K.. (2008). Banking on Basel: The Future of International Financial Regulation. Peterson Institute for International Economics, U.S.A.

[12] Van Dillen, J.G. (1964). History of the principal public banks.Frank Cass\& CO LTD.

[13] Von Neumann, J. (1945). First Draft of a Report on the EDVAC. Contract No. W-670-ORD-4926 between the United States Army Ordnance Department and the University of Pennsylvania.

[14] Wolff, R. W. (1981). Poisson arrivals see time averages, $<$ http://www2.isye.gatech.edu/ spyros/courses/IE7201/Fall-13/PASTA-proof.pdf >. [August 14, 2015].

[15] ***, (2015). Bank for International Settlements- Basel Committee on Banking Supervision, $<$ http://www.bis.org/bcbs/>. [August 10, 2015].

[16] ***, (2015). Erlang B Calculator, < http://home.earthlink.net/ malcolmhamer/Erlang-B.xls>. [August $14,2015]$.

[17] ***, (2010). The European Environment - State and Outlook 2010. European Environmental Agency.

[18] ***, (2015). European Banking Authority, <www.eba.europa.eu>. [September 02, 2015].

[19] ***, (2015). Kendall's notations, $<$ https://www.andrewferrier.com/oldpages/queueing_theory/Andy/kendall.html >. [August 10, 2015].

\section{ACKNOWLEDGEMENTS}

The authors would like to thank their families for support! 


\section{AUTHORS}

Florin-Catalin ENACHE graduated from the Faculty of Cybernetics, Statistics and Economic Informatics of the Academy of Economic Studies in 2008. Starting 2010 he holds a MASTER degree in the field of Economic Informatics, in the area of "Maximum Availability Architecture". His main domains of interest are: Computer Sciences, Database Architecture and Cloud Performance Management. Since 2014 he is a PhD. Candidate at the Bucharest University of Economic Studies, focusing his research on Performance management in Cloud environments.

Adriana-Nicoleta TALPEANU graduated from the Faculty of Cybernetics, Statistics and Economic Informatics of the Academy of Economic Studies in 2008. Starting 2010 she holds a MASTER degree in the field of Finance and Banking, in the area of "Managing Banking Systems". Her main domains of interest are: Banking Systems, Financial Regulations and Business Analysis. Since 2014 she is a $\mathrm{PhD}$. Candidate at the Bucharest University of Economic Studies, focusing her research on the effects of the prudential rules on the banking systems.
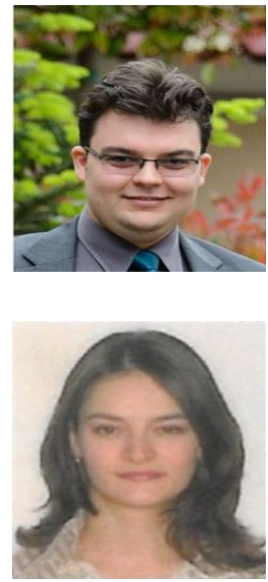